Pacific

Journal of

Mathematics

BOUNDARY REGULARITY OF HARMONIC MAPS

FROM HYPERBOLIC SPACE INTO NONPOSITIVELY CURVED MANIFOLDS

HAO YIN

Volume 232 No. 2

October 2007 


\title{
BOUNDARY REGULARITY OF HARMONIC MAPS FROM HYPERBOLIC SPACE INTO NONPOSITIVELY CURVED MANIFOLDS
}

\author{
HAO YIN
}

\begin{abstract}
This paper studies the boundary regularity of harmonic maps from hyperbolic space into manifolds with nonpositive curvature. Optimal results are obtained.
\end{abstract}

\section{Introduction}

Given two Riemannian manifolds $(M, g)$ and $(N, h)$ and a map $f$ from $M$ to $N$, the energy of $f$, if finite, is defined as

$$
E(f)=\int_{M} \frac{1}{2}|\nabla f|^{2} d V=\int_{M} \frac{1}{2} \operatorname{tr}_{g}\left(f^{*} h\right) d V .
$$

A map $f$ is said to be harmonic if it is an extremum of this energy functional. If $\left\{x_{i}\right\}$ and $\left\{y_{\alpha}\right\}$ are respectively local coordinates on $M$ and $N$, then the Euler-Lagrange equation of the energy functional $E(f)$ becomes

$$
\triangle f^{\alpha}+\Gamma_{\beta \gamma}^{\alpha}(f) \frac{\partial f^{\beta}}{\partial x_{i}} \frac{\partial f^{\gamma}}{\partial x_{j}} g^{i j}=0, \quad \alpha=1, \ldots, \operatorname{dim} N,
$$

where the sum's right members are the components of the tension field.

Eells and Sampson [1964] famously proved existence by the heat flow method for compact $M$ and $N$ when the sectional curvature of $N$ is nonpositive. Later, Liao and Tam [1992] generalized their work to complete noncompact $M$, with the condition that the total energy is finite. Li and Tam [1991] removed the finite energy condition; instead, they required the manifold satisfy some other geometric condition and that there exists some "approximate" harmonic map in that the $L^{p}$ norm of the square of the tension field is finite for $p>1$. In particular, without much effort, their results imply that if $M$ is hyperbolic space and $N$ has nonpositive sectional curvature, then, given any smooth $f: S^{m-1} \rightarrow N$, we can find a harmonic map $u$ from $M$ to $N$ with $f$ as the boundary value. For the precise statement, see Section 2.

MSC2000: primary 53C43; secondary 35J70.

Keywords: boundary regularity, harmonic map, hyperbolic space. 
If we take the hyperbolic space as the interior of the unit disk, there is the problem of boundary regularity, that is, how smooth could this $u$ be up to the boundary? This is essentially a problem of the boundary regularity of a uniformly degenerate elliptic system. For harmonic functions, complete results have been obtained in [Graham 1983]. Recently, Chruściel et al. [2005] applied the earlier work of Andersson and Chruściel [1996] to study the boundary regularity of an asymptotically hyperbolic Einstein metric and obtained optimal results. Common to all these results is that even if the boundary data is smooth, the solution may or may not be smooth, depending on whether the dimension of the domain is even or odd. In both cases, the solution has an expansion as a function of the defining function of the boundary. For even-dimensional hyperbolic space $M$, the formal calculation shows the expansion involves only power series, whose coefficients are determined by the given Dirichlet data and some Neumann data implicitly determined by the global properties. If the dimension of the hyperbolic space is odd, then the expansion involves terms like $\rho^{k}(\log \rho)^{l}$. Chruściel et al. [2005] showed, for a conformally Einstein metric, that this is the only obstruction to the boundary regularity, that is, the solutions are polyhomogeneous (see [Andersson and Chruściel 1996] and Section 3). The main result here shows the same phenomenon occurs for harmonic maps from hyperbolic space into a nonpositively curved manifold.

Theorem 1.1. Let $M=\mathbb{H}^{m}$ be a hyperbolic space and $N$ a complete manifold with nonpositive sectional curvature. For any smooth map $f: \partial M \rightarrow N$, the harmonic map $u$ with boundary value $f$ is polyhomogeneous up to the boundary. Moreover, $u$ has an expansion near the boundary whose coefficients can be determined by $f$ and some Neumann data. In particular, if $m$ is even, $u$ is smooth up to the boundary; if $m$ is odd, $u$ is $C^{m-2}$ up to the boundary and $C^{\infty}$ in the tangent direction of $\partial M$.

As remarked earlier, the existence and uniqueness of such boundary value problem follows trivially from the result of $\mathrm{Li}$ and Tam [1991]. A lemma in [Li and Tam 1993a] can be applied here to prove some partial regularity, that is, $C^{\beta}$ up to the boundary. To get the optimal results, we need the regularity theorem proved in [Andersson and Chruściel 1996]. Excepting those trivial conditions, there are two essential requirements: a good approximate solution and a regularity interval for the linearized operator. For the first, we need to construct the approximate solution and prove certain estimates. Since $f$ is now a map into a curved manifold instead of a section of a bundle, we have to do the construction locally and patch results together. The second requirement, in the case of [Chruściel et al. 2005], is discussed in great detail in [Lee 2006]. That discussion focused on a geometric operator, since the main application would be the Einstein metric. In our case, the 
linearization of the harmonic map equation is not a geometric operator. However, the main part of this linearization will be the Laplacian on hyperbolic space, which is discussed in [Lee 2006] and whose regularity interval is known [Andersson and Chruściel 1996; Lee 2006].

Following the idea of the proof of the main regularity theorem in [Andersson and Chruściel 1996], we will show the remaining terms in the linearization do not matter. We will rewrite their proof here, for these reasons: First, their regularity theorem is not in the exact form we need here, because the linearized operator is not a geometric one and because we will not directly study its regularity interval. Second, our proof will be simplified because our approximate solution is much better than they assumed, and we can do without many of their technical details.

The paper is organized as follows: In Section 2, we recall a result of $\mathrm{Li}$ and Tam to show existence and uniqueness. In Section 3, we will introduce the concept of polyhomogeneous and the Borel lemma. In Section 4, following the exposition of [Kichenassamy 2004] and using the Borel lemma, we show the existence of an approximating solution. In Section 5, some partial regularity is proved. In the final section, we prove Theorem 1.1.

Finally, we remark on [Li and Tam 1993a]. There, they discussed the existence, uniqueness and regularity of proper harmonic maps between hyperbolic spaces. Let $\mathbb{U}^{m}$ and $\mathbb{U}^{n}$ be hyperbolic spaces of dimension $m$ and $n$. The hyperbolic space $\mathbb{H}^{m}$ is identified with $\mathbb{D}^{m}=\left\{x \in \mathbb{R}^{m}|| x \mid<1\right\}$ with the Poincaré metric

$$
d s_{M}^{2}=4\left(1-\rho^{2}\right)^{-2}\left(d \rho^{2}+\rho^{2} \sum_{i, j=2}^{m} g_{i j} d \eta^{i} \eta^{j}\right),
$$

where $\rho^{2}=\sum_{i=1}^{m}\left(x^{i}\right)^{2}$ and $\sum_{i, j=2}^{m} g_{i j} d \eta^{i} d \eta^{j}$ is the standard metric on the unit sphere $S^{m-1}$. (In this paper, the hyperbolic metric is expressed in a different way.) Similarly, we identify $\mathbb{H}^{n}$ with $\mathbb{D}^{n}$ in $\mathbb{R}^{n}$ with the Poincaré metric

$$
d s_{N}^{2}=4\left(1-r^{2}\right)^{-2}\left(d r^{2}+r^{2} \sum_{p, q=2}^{n} h_{p q} d \theta^{p} d \theta^{q}\right),
$$

where $r^{2}=\sum_{p=1}^{n}\left(u^{p}\right)^{2}$ and $\sum_{p, q=2}^{n} h_{p q} d \theta^{p} d \theta^{q}$ is the standard metric on the unit sphere $S^{n-1}$. A map $u$ from $\mathbb{H}^{m}$ to $\mathbb{H}^{n}$ is now given by $r\left(\rho, \eta^{i}\right)$ and $\theta^{p}\left(\rho, \eta^{i}\right)$. Calculations show [Li and Tam 1993a, Section 1] the tension field is now

$$
\begin{aligned}
\tau(u)^{1}=\frac{\left(1-\rho^{2}\right)^{2}}{4} \Delta_{0} r+\frac{1}{4}\left(2(m-2)\left(1-\rho^{2}\right) \rho \frac{\partial r}{\partial \rho}\right. \\
\left.+\frac{r\left(1-\rho^{2}\right)^{2}\left(2\left|\nabla_{0} r\right|^{2}-\left(1+r^{2}\right) \sum_{p, q=2}^{n} h_{p q}\left\langle\nabla_{0} \theta^{p}, \nabla_{0} \theta^{q}\right\rangle\right)}{1-r^{2}}\right),
\end{aligned}
$$


and

$$
\begin{aligned}
\tau(u)^{s}=\frac{\left(1-\rho^{2}\right)^{2}}{4}\left(\triangle_{0} \theta^{s}\right. & \left.+\sum_{p, q=2}^{m} \tilde{\Gamma}_{p q}^{s}\left\langle\nabla_{0} \theta^{p}, \nabla_{0} \theta^{q}\right\rangle\right) \\
& +\frac{1-\rho^{2}}{2}\left((m-2) \rho \frac{\partial \theta^{s}}{\partial \rho}+\frac{\left(1+r^{2}\right)\left(1-\rho^{2}\right)\left\langle\nabla_{0} r, \nabla_{0} \theta^{2}\right\rangle}{r\left(1-r^{2}\right)}\right),
\end{aligned}
$$

for $s \geq 2$. Here, $\Delta_{0}$ is the Euclidean Laplacian, $\nabla_{0}$ is the Euclidean gradient, and $\tilde{\Gamma}_{p q}^{s}$ are the Christoffel symbols for the standard metric on $S^{n-1}$.

Compared to Equation (1), the $1-r^{2}$ in the denominator causes more difficulty since $\lim _{\rho \rightarrow 1} r=1$. In this case, uniqueness of the boundary value problem is not true without further assumptions. There are known examples, see [ $\mathrm{Li}$ and Tam 1993a], showing that one can't expect the harmonic map to be $C^{1}$ even if the boundary map is analytic. Li and Tam showed that if we further assume the harmonic map is $C^{1}$ up to the boundary, then the uniqueness is true. Moreover, under this assumption, if the boundary map is $C^{k, \alpha}$ for $1 \leq k \leq m-1,0<\alpha \leq 1$ and has nowhere vanishing energy density, then the harmonic map will be $C^{k, \gamma}$ for $0<\gamma<\alpha$ up to the boundary.

Their result should be compared with Section 5, where we use a lemma of theirs. A further question is: if we assume that the boundary map is smooth with nowhere vanishing energy density and the harmonic map is $C^{1}$ up to the boundary, what's the optimal regularity? (See problems in [Li and Tam 1993b].) In particular, will the harmonic map be smooth in the tangential direction? To answer this, we tried the method in this paper. We can show, under the above assumptions, that there is an approximate solution whose expansion coefficients are determined by boundary values and Neumann data. However, it's not known how to show the (unique) harmonic map is plurihomogeneous and has such an expansion.

\section{Existence and uniqueness}

The results in this section are all known.

Theorem 2.1 [Li and Tam 1991, Theorem 5.2]. Let $M^{m}$ be a complete manifold with Ricci curvature bounded below by $-K \leq 0$, and $\inf _{x \in M} V_{x}(1)=b>0$. Assume that the lower bound of the spectrum $\lambda(M)$ of $M$ is positive. Let $N^{n}$ be a complete manifold with nonpositive curvature. Let $w \in C^{1}(M, N)$ such that the energy density of $w$ is bounded and the square of the norm of the tension field of $w$ is bounded and in $L^{p}(M)$ for some $p>1$. Then there exists a unique solution of the harmonic map flow $u: M \times(0, \infty) \rightarrow N$ such that

(i) $u(0)=w$.

(ii) $u_{\infty}(x)=\lim _{t \rightarrow \infty} u(\cdot, t)$ is a harmonic map. 
(iii) $\sup _{M} e\left(u_{\infty}\right)<\infty$.

(iv) $\sup _{x \in M} \operatorname{dist}\left(u_{\infty}(x), w(x)\right)<\infty$.

(v) If, in addition, the norm of the tension field of $w$ tends to 0 as $x \rightarrow \infty$, then $\lim _{x \rightarrow \infty} \operatorname{dist}\left(u_{\infty}(x), w(x)\right)=0$.

From now on, $M$ will be $\mathbb{H}^{m}$ with the metric

$$
g_{M}=d r^{2}+\sinh ^{2} r d \theta^{2}=\frac{1}{\rho^{2}}\left(d \rho^{2}+\frac{\left(1-\rho^{2}\right)^{2}}{4} h_{0}\right),
$$

where $\rho=e^{-r}, r$ is the distance to some point in $M$, and $h_{0}$ is the standard metric on the unit sphere. It's well known that $M$ satisfies all geometric conditions of the above theorem. From time to time, we will take $\left(M, \partial M, \rho^{2} g_{M}\right)$ as a Riemannian manifold with boundary. This is known as the conformal compactification. Assume we have a smooth map $f: \partial M \rightarrow N$. To use the above theorem, we need to construct $w: \bar{M} \rightarrow N$ satisfying all the assumptions. Assume that $f$ is homotopic to the trivial map, which is always true if $\operatorname{dim} M \geq 3$. Let $w: \bar{M} \rightarrow N$ be a smooth extension of $f$. Combining $\rho$ with a coordinate system $\left\{\theta_{i}\right\}_{i=1}^{m-1}$ on an open set of $\partial M$ gives a coordinate system near the boundary. Collectively, we write them as $x_{i}$, where $x_{1}=\rho$. Then the energy density of $w$ is

$$
e(w)=\frac{1}{2} g_{M}^{i j} \frac{\partial w^{\alpha}}{\partial x_{i}} \frac{\partial w^{\beta}}{\partial x_{j}} h_{\alpha \beta} .
$$

Since $g^{i j}=O\left(\rho^{2}\right)$ and all other terms are bounded, we know $w$ has finite energy density. The tension field in these coordinates is

$$
\begin{aligned}
\tau(w)^{\alpha}=\rho^{2} \frac{\partial^{2} w^{\alpha}}{\partial \rho^{2}}-(m-2) \rho \frac{\partial w^{\alpha}}{\partial \rho}-2(m & -1) \frac{\rho^{3}}{1-\rho^{2}} \frac{\partial w^{\alpha}}{\partial \rho} \\
& +\frac{4 \rho^{2}}{\left(1-\rho^{2}\right)^{2}} \triangle_{0} w^{\alpha}+\Gamma_{\beta \gamma}^{\alpha} \frac{\partial w^{\beta}}{\partial x_{i}} \frac{\partial w^{\gamma}}{\partial x_{j}} g^{i j},
\end{aligned}
$$

where $\triangle_{0}$ is the Laplacian for $h_{0}$. Therefore $|\tau(w)|^{2}=O\left(\rho^{2}\right)$; it is in $L^{p}$ for $p$ sufficiently large and decays to zero at infinity. Theorem 2.1 gives us a harmonic map $u: M \rightarrow N$ with $f$ as the boundary value.

Proposition 2.2. This $u$ is uniquely determined by $f$.

The proof is the maximum principle plus a well-known calculation of Schoen and Yau [1979]. See also [Li and Tam 1991]. Because of this uniqueness theorem, it suffices to prove regularity for one harmonic map satisfying the boundary condition, in particular, the one constructed in Section 4. 


\section{Polyhomogeous maps and the Borel lemma}

The lemmas in this section will help construct the approximate solution in the next section. Andersson and Chruściel [1996] define the polyhomogeous function. It will suffice for our problem to use a simpler definition that is a special case of theirs. Let $f$ be a function defined on $U \times[0, \epsilon)$, where $U$ is an open subset of $\partial M$. We will use $(\theta, \rho)$ as coordinates in $U \times[0, \epsilon)$, where $\theta$ stands for the collective $\theta_{i}$.

Definition 3.1. A smooth function $u$ on $U \times(0, \epsilon)$ is said to be polyhomogeous if there exist smooth functions $a_{i j}(\theta)$ such that, for any integers $p>0$ and $q>0$, there exists $N>0$ for which

$$
\left|D^{\alpha}\left(u-\sum_{i=0}^{N} \sum_{j=0}^{i} a_{i j} \rho^{i}(\log \rho)^{j}\right)\right| \leq C(p, q) \rho^{q},
$$

where $\alpha$ is a multiindex of order $|\alpha| \leq p$ and $D^{\alpha}$ means partial differential (governed by $\alpha$ ) in the $\theta_{i}$ and $\rho$. We call $a_{i j}(\theta)$ the coefficients of the expansion of $u$.

According to the definition, $u$ is $C^{\infty}$ in $\theta$ when $\rho=0$. If there is no log term for $i=1, \ldots, k$, then $u$ is $C^{k}$ up to the boundary. If $u: U \times[0, \epsilon) \rightarrow N$ is a map, we assume that the image lies in a neighborhood with coordinates $\left\{y^{\alpha}\right\}$. If $u^{\alpha}$ is polyhomogeous, we would like to say that the map $u$ is polyhomogenous. To justify the definition, we need the following lemma, whose form is more general than what we need here but will be useful in Section 4. For simplicity, we consider only the composition of scalar functions.

Lemma 3.2. Let $u$ be a polyhomogeneous function defined on $U \times[0, \epsilon)$. Suppose $F(t, \theta)$ defined on $\mathbb{R} \times U$ is a smooth function such that $\partial^{\alpha} F(t, \theta)$ is bounded. Then $F(u(\rho, \theta), \theta)$ is also polyhomogeneous, and the coefficients of expansion can be calculated formally by plugging the expansion of $u$ into the Taylor series of $F$ with respect to $t$.

Proof. We can assume without loss of generality that $u(0, \theta)=0$ for all $\theta$. If otherwise, we consider $F^{\prime}(u, \theta)=F(u-u(0, \theta), \theta)$ instead of $F$. Recall Taylor's formula for $F$ with integral remainder $R$ :

$$
\begin{aligned}
& F(u, \theta)-F(0, \theta)-\cdots-\frac{1}{N !} u^{N} F^{(N)}(0, \theta)= \\
& \quad \int_{0}^{u} \int_{0}^{x_{1}} \cdots \int_{0}^{x_{N}} F^{(N+1)}\left(x_{N+1}, \theta\right) d x_{N+1} \cdots d x_{1}=: R .
\end{aligned}
$$

For multiindex $|\alpha|<m$, we need to estimate $\partial^{\alpha} R$. Let $\alpha_{i}$ and $\alpha^{\prime}$ be multiindices such that $\alpha_{1}+\alpha_{2}+\cdots+\alpha_{s}+\alpha^{\prime}=\alpha$ and $\alpha^{\prime}$ involves only $\theta$ derivatives. A typical 
term in $\partial^{\alpha} R$ looks like

$$
\partial^{\alpha_{1}} u \cdots \partial^{\alpha_{s}} u \int_{0}^{u} \cdots \int \partial^{\alpha^{\prime}} F^{(N+1)}\left(x_{N+1}, \theta\right) d x_{N+1} \cdots d x_{1+s} .
$$

Since $u$ is polyhomogeneous, $\left|\partial^{\alpha_{i}} u\right| \leq C \rho^{-\left|\alpha_{i}\right|}$. If $N>m+n$, using the assumption that $\partial^{\alpha^{\prime}} F^{(N+1)}$ is bounded,

$$
\left|\partial^{\alpha} R\right| \leq C \rho^{n},
$$

where we have also used that $u(0, \theta)=0$. According to the definition of polyhomogeneous, we can choose $N$ such that

$$
u(\rho, \theta)=\sum_{i=1}^{N} \sum_{j=0}^{i} a_{i j}(\theta) \rho^{i}(\log \rho)^{j}+o(m, n+m),
$$

where $o(m, n+m)$ satisfies

$$
\left|\partial^{\alpha} o(m, n)\right| \leq C \rho^{n+m}, \quad \text { for }|\alpha|<m
$$

The summation starts from $i=1$ because we assumed $u(0, \theta)=0$. Plug this into the left hand side of the Taylor formula. Collect those terms $\rho^{i}(\log \rho)^{j}$ with $i \leq N$ and with no $o(m, m+n)$ factor. This is we expect from a formal calculation, and we will make this the first $N$ levels in the expansion of $F(u(\rho, \theta), \theta)$. The lemma follows because the remaining terms have partial derivatives that decay like $\rho^{n}$.

The next lemma, [Andersson and Chruściel 1996, Lemma 3.1.2], is a generalization of the Borel lemma as given in [Hörmander 1983, Chapter 1]. It serves as a bridge from the formal solution to an approximate solution. In other words, the Borel lemma and the definition of a polyhomogeneous function make formal calculations approximately true.

Lemma 3.3. Given a family of smooth functions $a_{i j}(\theta)$ defined on $U$, we have a polyhomogeneous function $u$ defined on $U \times[0, \epsilon)$ with expansion coefficients $a_{i j}$.

\section{Formal solution and approximate solution}

The derivation of the formal solution follows [Kichenassamy 2004]. We start locally. For $p \in \partial M$, let $(\theta, \rho)$ be a coordinate system near $p$ as before. Let $\left\{y^{\alpha}\right\}$ be a coordinate system around $f(p)$. Setting $D=\rho \partial / \partial \rho$, the harmonic map equation $\tau(u)=0$ can be written as

$$
D(D-m+1) u^{\alpha}+\rho^{2} \Gamma_{\beta \gamma}^{\alpha}(u) \frac{\partial u^{\beta}}{\partial \rho} \frac{\partial u^{\gamma}}{\partial \rho}+\rho^{2} F\left(\rho \frac{\partial u}{\partial \rho}, \frac{\partial u}{\partial \theta}, \frac{\partial^{2} u}{\partial \theta^{2}}, u\right)=0,
$$


where $F$ is a smooth function of its arguments. We confine ourselves to the formal solution of the form (we drop the superscript $\alpha$ for simplicity)

$$
u(\theta, \rho)=\sum_{i=0}^{\infty} \sum_{j=0}^{i} a_{i j}(\theta) \rho^{i}(\log \rho)^{j} .
$$

The log term appears frequently in Fuchsian type equations and has a long history. The coefficients $a_{i j}(\theta)$ are smooth functions defined on a subset of $\partial M$ or $\partial M$ itself. We call

$$
\sum_{j=0}^{i} a_{i j}(\theta) \rho^{i}(\log \rho)^{j}
$$

the $i$-level part of the formal series. It's easy to check that for some integer $a$, the operator $D-a$ acts on the $i$-level part and satisfies:

(i) $D-a$ maps the $i$-level part to $i$-level part.

(ii) If $a \neq i$, for any $i$-level part $g$, the equation $(D-a) u=g$ determines $u$. Moreover, if $g$ has no log term, neither has $u$.

(iii) If $a=i$, for any $i$-level part $g$, the equation $(D-a) u=g$ determines $u$ except for the coefficient $a_{i 0}(\theta)$ of $u$. Moreover, if $g$ has no log term, $u$ will have a $\rho^{i}(\log \rho)$ term, whose coefficient is determined by $g$.

With these basic properties of $D-a$, we can examine Equation (2) level-bylevel. Assume $m>2$. For the 0 -level, we have $a_{00}(\theta)$ given by the boundary value $f$. For the 1-level, we know the 1-level of $u$ is zero. For higher level $k$, we would like to show the $k$-level of the last two terms in Equation (2) is determined by lower levels of $u$. Since $F$ is smooth, $F\left(\rho \partial u / \partial \rho, \partial u / \partial \theta, \partial^{2} u / \partial \theta^{2}, u\right)$ has a formal expansion, the $(k-2)$-level coefficients are determined by those of $l$-level of $u$ for $l \leq k-2$. It is easy to see that $\rho^{2} \Gamma_{\beta \gamma}^{\alpha}(u) \frac{\partial u^{\beta}}{\partial \rho} \frac{\partial u^{\gamma}}{\partial \rho}$ involves at most the $(k-1)$-level of $u$.

By induction, we can obtain a formal series $u$. All coefficients below level $m-1$ are determined by the boundary value only. Those above the $(m-1)$-level are determined by the boundary value and an arbitrary choice of $a_{m-1,0}(\theta)$. This arbitrariness can be regarded as the Neumann boundary condition and plays a subtle role in the theory of the regularity. For a solution, these Neumann boundary data are determined by global information.

More information can be obtained from the induction process. In fact, parity tells us that all odd levels below $m-1$ are zero. If $m$ is even, that is, $m-1$ is odd, there is no log term introduced when computing the $(m-1)$-level. Since this is the only chance of introducing a log term, the formal series will be a power series. If $m$ is odd, that is, $m-1$ is even, whether there is a log term depends on the initial value. A similar phenomenon is called a compatibility condition by $\mathrm{Li}$ and Tam. 
For $m=2$, it's easy to check the above discussion is also true.

Theorem 4.1. For a given smooth map $f: U \rightarrow N$ such that $f(U)$ lies in a coordinate neighborhood, there is a formal solution $u$ in the form of (3) to (2). All the coefficients are determined by boundary value $a_{00}$ and arbitrary Neumann data $a_{m-1,0}$. If $m$ is even, the series contains no $\log$ term.

By the Borel lemma, we have a polyhomogeneous map $u$ defined on a neighborhood of $p$.

Lemma 4.2. The map $u$ defined above is an approximate solution of (2) in that

$$
|\tau(u)| \leq C(k) \rho^{k} \quad \text { for any } k .
$$

Proof. From the definition, $\rho \partial u / \partial \rho, \partial u / \partial \theta$ and $\partial^{2} u / \partial \theta^{2}$ are all polyhomogeneous functions, whose expansion coefficients can be calculated formally. The proof follows from combining this fact with Lemma 3.2.

Remark 4.3. Although this approximate solution depends on a choice of coordinates around $f(p),|\tau(u)| \leq C \rho^{k}$ is coordinate free. Therefore, if were to choose another coordinate system $\left\{z^{\alpha}\right\}$ around $f(p)$ and express $u$ in the $z^{\alpha}$, then the expansion coefficients of $u^{\alpha}$ would agree with formal calculations in this coordinate system. The Dirichlet data remain the same; however, the Neumann data, that is, the coefficients of $\rho^{m-1}$, are transformed in a complicated way.

To prove our main theorem, we need a global approximate solution defined on $\partial M \times[0, \epsilon)$. Assume that we have defined an approximate solution $u$ on $K \times[0, \epsilon)$ for some open subset $K \subset \partial M$. We will show that we can make $K$ larger. For any $p \in \partial K$, take a small neighborhood $U$ of $p$ so that $f(U)$ lies in a coordinate neighborhood of $N$, and let $\left\{y^{\alpha}\right\}$ be the coordinates. The size of this $U(p)$ could be bounded from below by a positive constant depending on $N$ and $f$.

By the above discussion, we have an approximate solution $v$ on $U \times[0, \epsilon)$ with the prescribed Dirichlet boundary value on $U$. To have any chance of glueing $u$ and $v$ together, we must require the Neumann data of $u$ and $v$ agree on $K \cap U$.

To achieve this goal, note that $u$ is also an approximate solution when restricted to $K \cap U$. Therefore, we can write the expansion in the coordinates $y^{\alpha}$. Now the Neumann data are just $n$ smooth functions $a_{m-1,0}^{\alpha}(\theta)$ defined on $K \cap U$. Extend them arbitrarily to $U$, still denoting them by $a_{m-1,0}^{\alpha}$. With this set of Neumann data and the prescribed Dirichlet boundary data, we obtain as above a formal solution in the coordinates $y^{\alpha}$. Finally, the Borel lemma and Lemma 4.2 give an approximate solution $v$ on $U \times[0, \epsilon) . u$ and $v$ are not necessarily the same even on the intersection $(K \cap U) \times[0, \epsilon)$. However, they do have the same expansion on $(K \cap U) \times[0, \epsilon)$ in the coordinates $y^{\alpha}$. In fact, Lemma 3.2 shows this is true for any other coordinate system. 
To glue them together, we use an averaging argument. Before we can do that, we need to transform the map into a linear object. So consider the bundle $f^{*}(T N)$. Let $\xi(\theta, \rho)$ be a smooth family of sections of $f^{*}(T N)$ parametrized by $\rho$. Define

$$
F(\xi(\theta, \rho))=\exp _{f(\theta)} \xi(\theta, \rho),
$$

where exp is the exponential map on $N$. Conversely, we have

$$
F^{*}(q, \theta)=\exp _{f(\theta)}^{-1} q \in f^{*}(T N)_{\theta},
$$

where $q \in N$ is a point near $f(\theta)$.

Let $\left\{e_{i}\right\}$ be a locally frame of $f^{*}(T N)$ and $\left\{y^{\alpha}\right\}$ a coordinate system of $N$, and express everything in components. $F$ and $F^{*}$ are smooth maps from $\mathbb{R}^{n} \times U \rightarrow \mathbb{R}^{n}$. Lemma 3.2 says that if $u$ is a polyhomogeneous map, then $F^{*}(u(\theta, \rho), \theta)$ is a polyhomogeneous section.

Recall that $p \in \partial K$. Take open sets $V_{1}$ and $V_{2}$ such that $p \in V_{1} \subset \bar{V}_{1} \subset V_{2} \subset$ $\bar{V}_{2} \subset U$ and the size of $V_{1}$ can be bounded uniformly from below. Let $a, b$ be nonnegative smooth functions defined on $K \cap U \cup V_{1}$ such that

(i) $a=1$ on $K \cap U \backslash V_{2}$;

(ii) $b=1$ on $V_{1}$;

(iii) $a+b=1$ on $K \cap U \cup V_{1}$.

Let $\xi_{u}$ be the section $F^{*}(u, \theta)$ on $K \cap U$ and $\xi_{v}$ be the section $F^{*}(v, \theta)$ on $U$. Their expansions are the same on $K \cap U$. On $K \cap U \cup V_{1}$, define $\xi=a \xi_{u}+b \xi_{v}$. It follows from the definition of $a$ and $b$ that

(i) $\xi$ agrees with $\xi_{u}$ on $K \cap U \backslash V_{2}$;

(ii) $\xi$ agrees with $\xi_{v}$ on $V_{1}$;

(iii) the expansion of $\xi$ agrees with $\xi_{u}$ on $K \cap U$ and agrees with $\xi_{v}$ on $K \cap U \cup V_{1}$.

Now set $w=F(\xi, \theta)$. Lemma 3.2 ensures that $w$ is a polyhomogenous map and $w$ is an approximate solution, since its expansion agrees with $u$ or $v$. $w$ can be set to $u$ on $K \backslash U$. Now $w$ is an approximate solution defined on $K \cup V_{1}$. Set $K=K \cup V_{1}$ and repeat the above construction for other points on $\partial K$ until $K=\partial M$. Therefore, we have proved:

Theorem 4.4. Let $f$ be any smooth map from $\partial M$ to $N$, and assume that $f$ is homotopically trivial (which is always true for $m>2$ ). Then we can construct a map u from $M$ to $N$ such that

$$
|\tau(u)| \leq C(k) \rho^{k} \quad \text { for any } k .
$$

Proof. The above construction defines $u$ on $\partial M \times[0, \epsilon)$. Extend it arbitrarily to the interior. 


\section{Lower order regularity}

From now on, denote the approximate solution by $u_{0}$. It follows from the construction and the definition of polyhomogeneous functions that $u_{0}$ is at least $C^{1}$ up to the boundary. A calculation similar that in to Section 2 shows $u_{0}$ has bounded energy density. Theorem 2.1 shows that we have a solution $u(t)$ to the heat flow equation and $u(t)$ converges to a harmonic map $u_{\infty}$ from $M$ to $N$ such that $u_{\infty}$ has finite energy density and $\lim _{\rho \rightarrow 0} \operatorname{dist}\left(u_{\infty}(\theta, \rho)-u_{0}(\theta, \rho)\right)=0$.

The main result of this section will improve the last statement. Precisely, if we embed $N$ into some $\mathbb{R}^{K}, u_{0}, u(t)$, and $u_{\infty}$ can be taken as vector-valued functions. Denote by $A$ the second fundamental form of $N$ as a submanifold in $\mathbb{R}^{K}$. It's well known that the harmonic map equation can be written

$$
\triangle u+A(u)(d u, d u)=0 .
$$

Theorem 5.1. There exists some $\beta>0$ such that

$$
\left|(\rho \partial)^{\alpha}\left(u_{\infty}-u_{0}\right)\right| \leq C(k) \rho^{\beta},
$$

where $\alpha$ is a multiindex with order $|\alpha|=k$ and $\partial$ means partial derivatives in the $\theta_{i}$ and $\rho$.

The proof takes two steps. First, we establish the decay in the $C^{0}$ norm. Fix any point $o \in M$. Recall:

Lemma 5.2 [Li and Tam 1993a, Lemma 3.1]. For $0<s \leq m-1,(2 \rho /(1+\rho))^{s}$ is a superharmonic function when $\rho \neq 1$.

Remark 5.3. Li and Tam write the hyperbolic metric as $4\left(d \rho^{2}+\rho^{2} h_{0}\right) /\left(1-\rho^{2}\right)^{2}$. Therefore their $\rho$ is our $(1-\rho) /(1+\rho)$.

We present a modified and weaker version of [Li and Tam 1993a, Lemma 3.2]:

Lemma 5.4. There exists some positive $\beta>0$ such that the distance between $u_{0}$ and $u_{\infty}$ satisfies

$$
\operatorname{dist}\left(u_{0}(\theta, \rho), u_{\infty}(\theta, \rho)\right) \leq C \rho^{\beta},
$$

that is, as a vector valued function,

$$
\left|u_{0}(\theta, \rho)-u_{\infty}(\theta, \rho)\right| \leq C \rho^{\beta} .
$$

Proof. Li and Tam [1991] proved

$$
\left\|u_{t}\right\|^{2} \leq C e^{-\alpha t} .
$$

Since the sectional curvature of $N$ is nonpositive, a result of Hartman [1967] shows

$$
\left(\partial_{t}-\triangle\right)\left\|u_{t}\right\|^{2} \leq 0 .
$$


The initial value satisfies

$$
\left.\left\|u_{t}\right\|^{2}\right|_{t=0}=\left\|\tau\left(u_{0}\right)\right\|^{2} \leq C \rho^{k} \quad \text { for any } k .
$$

An application of maximum principle and Lemma 5.2 shows

$$
\left\|u_{t}\right\|^{2} \leq C \rho^{m-1}
$$

near the boundary. Therefore

$$
\left\|u_{t}\right\| \leq C e^{-\frac{\alpha}{4} t} \rho^{(m-1) / 4}
$$

and

$$
\operatorname{dist}\left(u_{0}, u_{\infty}\right) \leq \int_{0}^{\infty}\left\|u_{t}\right\| d t \leq C \rho^{(m-1) / 4} .
$$

To estimate higher order derivatives, we consider interior estimates on balls of uniform size. Around $p=\left(\rho^{\prime}, \theta^{\prime}\right) \in M$, define a coordinate system $\left\{x_{i}\right\}$ by

$$
\left(x_{1}, x_{2}, \ldots, x_{m}\right)=\left(\rho^{\prime}+\frac{1}{2} \rho^{\prime} x_{1}, \theta_{i}^{\prime}+\frac{1}{2} \rho^{\prime} x_{i}\right) .
$$

Let $U(p)=\left\{\left(x_{1}, \ldots, x_{m}\right) \mid \sum x_{i}^{2}<1\right\}$. In the $\left\{x_{i}\right\}$, the hyperbolic metric is

$$
\frac{\left(\rho^{\prime}\right)^{2}}{4 \rho^{2}}\left(d x_{1}^{2}+\frac{\left(1-\rho^{2}\right)^{2}}{4} \bar{g}^{i j} d x_{i} d x_{j}\right)
$$

where $\bar{g}^{i j} d \theta_{i} d \theta_{j}=h_{0}$. Similar coordinates are called Möbius coordinates in [Lee 2006]. Since $\rho \in\left(\frac{1}{2} \rho^{\prime}, \frac{3}{2} \rho^{\prime}\right)$, the coordinates have the advantage that, first, the metric components are comparable to $\delta^{i j}$ and, second, all their partial derivatives are bounded. This fact is essential for obtaining uniform estimates.

$u_{\infty}$ is a harmonic map; thus

$$
\triangle u_{\infty}+A\left(u_{\infty}\right)\left(d u_{\infty}, d u_{\infty}\right)=0 .
$$

$u_{0}$ is an approximate solution, so

$$
\triangle u_{0}+A\left(u_{0}\right)\left(d u_{0}, d u_{0}\right)=o\left(\left(\rho^{\prime}\right)^{\infty}\right),
$$

where $o\left(\left(\rho^{\prime}\right)^{\infty}\right)$ stands for some polyhomogeneous function with vanishing expansion coefficients, that is, any derivative of $o\left(\left(\rho^{\prime}\right)^{\infty}\right)$ decays as fast as one needs. Subtract (4) and (5) to get

$$
\begin{aligned}
\triangle\left(u_{0}-u_{\infty}\right)+A\left(u_{0}\right)\left(d\left(u_{0}-u_{\infty}\right), d u_{0}\right)+A\left(u_{0}\right)\left(d u_{\infty}, d\left(u_{0}-u_{\infty}\right)\right) \\
=\left(A\left(u_{0}\right)-A\left(u_{\infty}\right)\right)\left(d u_{\infty}, d u_{\infty}\right)+o\left(\left(\rho^{\prime}\right)^{\infty}\right) .
\end{aligned}
$$

We know that $u_{0}$ and $u_{\infty}$ both have finite energy density, that is, their first order derivatives are bounded (in these coordinates). Also $A$ is smooth such that

$$
\left|A\left(u_{0}\right)-A\left(u_{\infty}\right)\right| \leq C\left|u_{0}-u_{\infty}\right| .
$$


The interior $L^{p}$ estimate says, if $V(p)=\left\{\sum x_{i}^{2} \leq 1 / 2\right\}$,

$$
\left\|u_{0}-u_{\infty}\right\|_{W^{2, q}(V(p))} \leq C \sup _{U(p)}\left(\left|u_{0}-u_{\infty}\right|+\left|o\left(\left(\rho^{\prime}\right)^{\infty}\right)\right|\right) .
$$

Lemma 5.4 and Sobolev embedding gives

$$
\left\|u_{0}-u_{\infty}\right\|_{C^{1, \gamma}(V(p))} \leq C\left(\rho^{\prime}\right)^{\beta} .
$$

To apply the Schauder estimate for $\left\|u_{0}-u_{\infty}\right\|_{C^{l+2, \gamma}}$, we need two lemmas.

\section{Lemma 5.5.}

$$
\left\|u_{0}\right\|_{C^{l, \gamma}(U(p))} \leq C \quad \text { for any } l>0 .
$$

Proof. By the definition of the $x_{i}$, we know $\partial / \partial x_{i}$ is equivalent to $\frac{1}{2} \rho^{\prime} \partial / \partial \rho$ or $\frac{1}{2} \rho^{\prime} \partial / \partial \theta$. Therefore

$$
\frac{\partial^{|\alpha|}}{\partial^{\alpha_{1}} x_{1} \cdots \partial^{\alpha_{m}} x_{m}}=\left(\rho^{\prime} / 2\right)^{|\alpha|} \partial^{\alpha},
$$

where $\partial$ means $\partial / \partial \rho$ and/or $\partial \theta$. For $q \in U(p), \rho \in\left(\frac{1}{2} \rho^{\prime}, \frac{3}{2} \rho^{\prime}\right)$. Since $u_{0}$ is polyhomogeneous, $\left|\partial^{\alpha} u_{0}\right| \leq C \rho^{-|\alpha|}$. Hence, $\left(\rho^{\prime}\right)^{|\alpha|} \partial^{\alpha} u_{0}$ is bounded inside $U(p)$.

Corollary 5.6.

$$
\text { If } \begin{aligned}
\left\|A\left(u_{0}\right)\right\|_{C^{l, \gamma}(U(p))} & \leq C, \quad \text { for any } l>0, \text { and } \\
\left\|u_{0}-u_{\infty}\right\|_{C^{l, \gamma}(U(p))} & \leq C, \quad \text { then } \\
\left\|u_{\infty}\right\|_{C^{l, \gamma}(U(p))} & \leq C .
\end{aligned}
$$

\section{Lemma 5.7.}

$$
\left\|A\left(u_{0}\right)-A\left(u_{\infty}\right)\right\|_{C^{l, \gamma}(U(p))} \leq C\left\|u_{0}-u_{\infty}\right\|_{C^{l, \gamma}(U(p))} .
$$

Proof. To see this, extend the definition of $A$ smoothly to a neighborhood of $N$ in $\mathbb{R}^{K}$. When $u_{0}$ and $u_{\infty}$ are sufficiently close, the line segment $\gamma$ connecting them remains in this neighborhood. So $\gamma:[0,1] \rightarrow \mathbb{R}^{K}$ is given by

$$
\gamma(t)=(1-t) u_{0}+t u_{\infty}
$$

$\gamma$ depends implicitly on $q \in U(p)$.

$$
\begin{aligned}
A\left(u_{\infty}\right)-A\left(u_{0}\right) & =\int_{0}^{1} \frac{d}{d t} A(\gamma(t)) d t \\
& =\int_{0}^{1} D A\left((1-t) u_{0}+t u_{\infty}\right) \cdot\left(-u_{0}+u_{\infty}\right) d t
\end{aligned}
$$

These lemmas allow us to use Schauder estimates inductively, which concludes the proof of Theorem 5.1. 
Remark 5.8. Results in this section are interior estimates, that is, they hold on a ball of fixed size inside the hyperbolic space. Theorem 5.1 roughly says that the difference in any $C^{k}$ norm measured in geodesic ball between $u_{0}$ and $u_{\infty}$ decays at order $\beta$ as $\rho$ goes to 0 . This fact starts the next section.

\section{Full regularity}

Now we can prove the main Theorem 1.1. We need some more definitions. Following the convention of [Andersson and Chruściel 1996], we denote by $C_{k}$ the functions differentiable $k$ times. Set $M_{\delta}=\{(\rho, \theta) \mid \rho<\delta\}$ for some $0<\delta<1$.

Definition 6.1. A $C_{k}$ function on $M$ is in $C_{k}^{\alpha}(M)$ if this norm is finite:

$$
\|f\|_{C_{k}^{\alpha}(M)}=\|f\|_{C_{k}\left(M \backslash M_{\delta}\right)}+\sup _{0 \leq|\gamma| \leq k,(\rho, \theta) \in M_{\delta}} \rho^{-\alpha}\left|\partial^{\gamma} f\right| .
$$

Definition 6.2. A $C_{k}^{\alpha}(M)$ function is said to be in $C_{k+\lambda}^{\alpha}(M)$ if this norm is finite:

$$
\begin{aligned}
\|f\|_{C_{k+\lambda}^{\alpha}(M)}^{\alpha}=\|f\|_{C_{k}^{\alpha}(M)} & +\|f\|_{C_{k+\lambda}\left(\overline{M \backslash M_{\delta}}\right)} \\
& +\sup _{y \in M_{\delta},|\gamma|=k} \sup _{y^{\prime} \neq y, d_{H}(x, y) \leq 1} \rho^{-\alpha+k+\lambda} \frac{\left|\partial^{\gamma} f(y)-\partial^{\gamma} f\left(y^{\prime}\right)\right|}{\left|y-y^{\prime}\right|^{\lambda}},
\end{aligned}
$$

where $C_{k+\lambda}\left(\overline{M \backslash M_{\delta}}\right)$ is the ordinary Hölder norm on a compact set, $y=(\rho, \theta)$ and $y^{\prime}$ are points in $M_{\delta}, d_{H}$ is the hyperbolic distance, and $\partial$ is as before.

The definition means the $C^{k+\lambda}$ norm of $f$ measured on a geodesic ball $U(p)$ decays like $\rho^{\alpha}$ as $p$ moves towards the infinity. In these terms, Theorem 5.1 says

$$
u_{0}-u_{\infty} \in C_{k}^{\beta} \quad \text { for any } k>0 \text { and some } \beta>0 .
$$

Definition 6.3. For some $\mu \in(0,1]$, a function $f \in C_{k+\lambda}^{\alpha}(M)$ is said to be in $C_{k+\lambda, 0+\mu}^{\alpha}(M)$ if this norm is finite:

$$
\|f\|_{C_{k+\lambda, 0+\mu}^{\alpha}(M)}=\|f\|_{C_{k+\lambda}^{\alpha}(M)}+\sup _{|\gamma|=k, \theta \neq \theta^{\prime}} \rho^{-\alpha} \frac{\left|(\rho \partial)^{\gamma}\left(f(\rho, \theta)-f\left(\rho, \theta^{\prime}\right)\right)\right|}{\left|\theta-\theta^{\prime}\right|^{\mu}} .
$$

Definition 6.4. A function $f \in C_{k+\lambda, 0+\mu}^{\alpha}$ is said to be in $C_{k+\lambda, l+\mu}^{\alpha}$ if, for $0 \leq|\gamma| \leq l$, we have $\partial_{\theta}^{\gamma} f \in C_{\min (0, k+\lambda-|\gamma|), 0+\mu}^{\alpha}$. Define the norm to be

$$
\|f\|_{C_{k+\lambda, l+\mu}^{\alpha}(M)}=\|f\|_{C_{k+\lambda, 0+\mu}^{\alpha}(M)}+\sum_{0 \leq|\gamma| \leq l}\left\|\partial_{\theta}^{\gamma} f\right\|_{C_{\min (0, k+\lambda-|\gamma|), 0+\mu}^{\alpha}\left(M_{\delta}\right)} .
$$

Our starting point is a polyhomogeneous approximate solution $u_{0}$ and a harmonic map $u$ (for simplicity, we will henceforth use $u$ instead of $u_{\infty}$ ) such that, as 
given in Theorem $5.1, u_{0}-u \in C_{\infty}^{\beta}$. The harmonic map equation is

$$
\triangle u^{\alpha}+A^{\alpha}(u)\left(\frac{\partial u}{\partial x_{i}}, \frac{\partial u}{\partial x_{j}}\right) g^{i j}=0 .
$$

Here $\left(x_{1}, \ldots, x_{m}\right)=(\rho, \theta)$ and $g=\left(d \rho^{2}+\left(1-\rho^{2}\right)^{2} / 4 h_{0}\right) / \rho^{2}$.

More precisely,

$$
\Delta u^{\alpha}+A^{\alpha}(u)\left(\rho \frac{\partial u}{\partial \rho}, \rho \frac{\partial u}{\partial \rho}\right)+A^{\alpha}(u)\left(\rho \frac{\partial u}{\partial \theta_{i}}, \rho \frac{\partial u}{\partial \theta_{j}}\right) \bar{g}^{i j}=0
$$

where

$$
\triangle f=\rho^{2} \frac{\partial^{2} f}{\partial \rho^{2}}-(m-2) \rho \frac{\partial f}{\partial \rho}-2(m-1) \frac{\rho^{3}}{1-\rho^{2}} \frac{\partial f}{\partial \rho}+\frac{4 \rho^{2}}{\left(1-\rho^{2}\right)^{2}} \triangle_{0} f .
$$

Therefore, the equation has the form $F\left(\rho, u, \rho \partial u, \rho^{2} \partial^{2} u\right)=0$, or, for simplicity, $F[u]=0$.

Lemma 6.5. For any small $\epsilon>0$, we have $u_{0}-u \in C_{\infty, \infty}^{m-1-\epsilon}$.

Proof. Set $v=u-u_{0}$ and consider

$$
G[v]=F\left[u_{0}+v\right]-F\left[u_{0}\right]-F^{\prime}\left[u_{0}\right] v .
$$

To see the meaning of this, set $\phi(t)=F\left[u_{0}+t v\right]$ and consider the Taylor expansion

$$
\phi(1)-\phi(0)-\phi^{\prime}(0)=\int_{0}^{1} \int_{0}^{t} \phi^{\prime \prime}(s) d s d t .
$$

Hence,

$$
\begin{aligned}
\phi^{\prime}(0) & =F^{\prime}\left[u_{0}\right] v \\
& =\Delta v+2 A\left(u_{0}\right)\left(\frac{\partial v}{\partial x_{i}}, \frac{\partial u_{0}}{\partial x_{j}}\right) g^{i j}+D A\left(u_{0}\right)\left(\frac{\partial u_{0}}{\partial x_{i}}, \frac{\partial u_{0}}{\partial x_{j}}\right) g^{i j} v .
\end{aligned}
$$

Assume $F=F(\rho, z, p, w)$, where $p$ and $w$ abbreviate $p_{i}$ and $w_{i j}$. The partial derivatives below should be understood as a sum of many terms. Therefore

$$
\begin{aligned}
\phi^{\prime \prime}(s)=\partial_{z}^{2} F v^{2}+\partial_{p}^{2} F(\rho \partial v)^{2}+\partial_{w}^{2} F\left(\rho^{2} \partial^{2} v\right)^{2} & \\
& +\partial_{z p}^{2} F v(\rho \partial v)+\partial_{z w}^{2} F v\left(\rho^{2} \partial^{2} v\right)+\partial_{w p}^{2} F(\rho \partial v)\left(\rho^{2} \partial^{2} v\right) .
\end{aligned}
$$

All partial derivatives of $F$ are bounded. Since $v \in C_{\infty}^{\beta}$, so is $\rho \partial v$ and $\rho^{2} \partial^{2} v$. Hence $G[v]=\int_{0}^{1} \int_{0}^{t} \phi^{\prime \prime}(s) d s d t \in C_{\infty}^{2 \beta}$.

$u$ is a solution; so $F\left[u_{0}+v\right]=0$, and $u_{0}$ is an approximate solution, that is, $F\left[u_{0}\right] \in C_{\infty, \infty}^{\infty}$. Therefore $F^{\prime}\left[u_{0}\right] v \in C_{\infty}^{2 \beta}$. The linearized operator has the structure

$$
F^{\prime}\left[u_{0}\right] v=\triangle v+\rho B_{1}(\rho \partial v)+\rho^{2} B_{2} v,
$$


where $B_{1}$ and $B_{2}$ are polyhomogeneous coefficients.

We have shown (see Lemma 5.5) that polyhomogeneous functions lie in $C_{\infty}^{0}$, that is, all derivatives measured in a geodesic unit ball are bounded. So

$$
\rho B_{1}(\rho \partial v)+\rho^{2} B_{2} v \in C_{\infty}^{1+\beta} .
$$

Hence $\Delta v \in C_{\infty}^{\min (2 \beta, 1+\beta)}$.

According to [Lee 2006], $(0, m-1)$ is a regularity interval of the Laplacian operator as defined in [Andersson and Chruściel 1996]. Precisely, if $\min (2 \beta, 1+$ $\beta) \in(0, m-1)$, then

$$
v \in C_{\infty}^{\min (2 \beta, 1+\beta)} .
$$

Set $\beta=\min (2 \beta, 1+\beta)$, and repeat the argument until $\beta>1$. Now assume $1<$ $\beta<m-1$, and use the embedding

$$
v \in C_{\infty}^{\beta} \subset C_{\infty, 1}^{\beta-1}
$$

Set $\beta=\beta-1$, and repeat the above argument to get

$$
F^{\prime}\left[u_{0}\right] v \in C_{\infty, 1}^{2 \beta} .
$$

Now we claim that $v \in C_{\infty, 1}^{2 \beta}$. In fact, this is a direct consequence of [Andersson and Chruściel 1996, Theorem 4.1.3]. In that theorem, they discuss $L \in \mathrm{OP}_{C_{k+\lambda, l+\mu}^{0}}$. Recall the definition of $\mathrm{OP}_{C_{k+\lambda, l+\mu}^{0}}$ : if

$$
L=\sum_{|\alpha| \leq 2} a_{\alpha}(y)(\rho \partial)^{\alpha}
$$

then $L$ is said to be in class $\mathrm{OP}_{C_{k+\lambda, l+\mu}^{0}}$ if $a_{\alpha} \in C_{k+\lambda, l+\mu}^{0}$. It's easy to see $\triangle$ is in class $\mathrm{OP}_{C_{\infty, \infty}^{0}}$.

We can repeat the above argument to prove the lemma.

Remark 6.6. In fact, the argument above will not work for $m=2$. In this case, $\min (2 \beta, \beta+1)$ cannot be larger than 1 because $m-1=1$. The proof needs a modification involving an interpolation inequality. See [Andersson and Chruściel 1996, Lemma 5.1.3].

The above process stops at $v \in C_{\infty, \infty}^{m-1-\epsilon}$. We can't expect better results because we have used arbitrary Neumann data for constructing $u_{0}$. Now we take a closer look at the structure of Laplacian operator

$$
\Delta v=\rho^{2} \frac{\partial^{2} v}{\partial \rho^{2}}-(m-2) \rho \frac{\partial v}{\partial \rho}-2(m-1) \frac{\rho^{3}}{1-\rho^{2}} \frac{\partial v}{\partial \rho}+\frac{4 \rho^{2}}{\left(1-\rho^{2}\right)^{2}} \triangle_{0} v .
$$


Since $v \in C_{\infty, \infty}^{m-1-\epsilon}$, we know

$$
\rho^{2} \frac{\partial^{2} v}{\partial \rho^{2}}-(m-2) \rho \frac{\partial v}{\partial \rho} \in C_{\infty, \infty}^{m-\epsilon}
$$

This is a Fuchsian-type ordinary differential operator, with indicial roots

$$
\mu_{-}=0 \quad \text { and } \quad \mu_{+}=m-1 .
$$

Set

$$
\eta=\rho^{2} \frac{\partial^{2} v}{\partial \rho^{2}}-(m-2) \rho \frac{\partial v}{\partial \rho} .
$$

Then we have an explicit formula for $v$ :

$$
v=A \rho^{\mu_{-}}+B \rho^{\mu_{+}}+G_{0}(\eta)
$$

with

$$
G_{0}(\eta)=\frac{1}{\mu_{+}-\mu_{-}}\left\{\rho^{\mu_{+}} \int_{0}^{\rho} s^{-1-\mu_{+}} \eta(s) d s-\rho^{\mu_{-}} \int_{0}^{\rho} s^{-1-\mu_{-}} \eta(s) d s\right\} .
$$

See [Andersson and Chruściel 1996, (4.2.8)]. (This formula for $G_{0}(\eta)$ is true because $\eta=O\left(\rho^{\alpha}\right)$ with $\alpha>\mu_{+}$.)

Now, since we know $v \in C_{\infty, \infty}^{m-1-\epsilon}, A=0 . B$ is a function of $\theta$. In other words,

$$
v(\theta, \rho)=B(\theta) \rho^{m-1}+G_{0}(\eta) .
$$

Taking any $\rho$ small but nonzero, we find $B$ is smooth.

This property of $G_{0}$ is studied carefully in [Andersson and Chruściel 1996, Proposition 3.4.3]. From (3.4.25) in the same reference, we know that $G(\eta) \in$ $C_{\infty, \infty}^{m-\epsilon}$.

From here we take a slightly different approach from Andersson and Chruściel. We reconstruct the approximate solution, since we now have better knowledge of the Neumann data. The construction is the same as in Section 4, the only difference is that we can do the formal calculation globally. The reason is that $u$ is now a map from $M$ to $\mathbb{R}^{K}$, that is, a vector-valued function. We use the prescribed boundary data as the coefficient of $\rho^{0}$ and use the $\rho^{m-1}$ coefficient of $u$ plus $B(\theta)$ as the Neumann data. Denote bu $u_{1}$ the new approximate solution. Note that the image of $u_{1}$ may not lie on $N$. However, we will see that this is not a problem for the proof below. The construction process gives

$$
u_{1}=u_{0}+B(\theta) \rho^{m-1}+\sum_{i=m}^{\infty} \sum_{j=0}^{i} b_{i j} \rho^{i}(\log \rho)^{j} .
$$


Setting $v_{1}=u-u_{1}$, we have

$$
\begin{aligned}
v_{1} & =v-B(\theta) \rho^{m-1}-\sum_{i=m}^{\infty} \sum_{j=0}^{i} b_{i j} \rho^{i}(\log \rho)^{j} \\
& =G_{0}(\eta)-\sum_{i=m}^{\infty} \sum_{j=0}^{i} b_{i j} \rho^{i}(\log \rho)^{j} \in C_{\infty, \infty}^{m-\epsilon} .
\end{aligned}
$$

As before,

$$
F\left[u_{1}+v_{1}\right]-F\left[u_{1}\right]-F^{\prime}\left[u_{1}\right] v_{1}=G\left[u_{1}\right] v_{1} \quad \text { and } \quad G\left[u_{1}\right] v_{1} \in C_{\infty, \infty}^{2(m-\epsilon)} .
$$

Since $F\left[u_{1}+v_{1}\right]=0$ and $F\left[u_{1}\right] \in C_{\infty, \infty}^{\infty}$, we have $F^{\prime}\left[u_{1}\right] v_{1} \in C_{\infty, \infty}^{2(m-\epsilon)}$. The structure of $F^{\prime}\left[u_{1}\right]$ is the same as $F^{\prime}\left[u_{0}\right]$ and $u_{1}$ is polyhomogeneous as is $u_{0}$. Hence

$$
\triangle v_{1} \in C_{\infty, \infty}^{m+1-\epsilon} .
$$

$m+1-\epsilon$ is not in the regularity interval of $\triangle$, so we write the Laplacian to see

$$
\rho^{2} \frac{\partial^{2} v_{1}}{\partial \rho^{2}}-(m-2) \rho \frac{\partial v_{1}}{\partial \rho} \in C_{\infty, \infty}^{m+1-\epsilon} .
$$

Letting $\eta_{1}=\rho^{2} \partial^{2} v_{1} / \partial \rho^{2}-(m-2) \rho \partial v_{1} / \partial \rho, v_{1}$ can be given explicitly by $v_{1}=$ $A+B \rho^{m-1}+G_{0}\left(\eta_{1}\right)$. Since we have $v_{1} \in C_{\infty, \infty}^{m-\epsilon}$, both $A$ and $B$ are zero. [Andersson and Chruściel 1996, Proposition 3.4.3] says $v_{1} \in C_{\infty, \infty}^{m+1-\epsilon}$. Repeating the above argument, we can show $u-u_{1}=v_{1} \in C_{\infty, \infty}^{\infty}$.

This finishes Theorem 1.1, since $u$ is polyhomogenous and has the same expansion coefficients as $u_{1}$. The "moreover" part of the theorem follows from a formal calculation.

\section{Acknowledgement}

The author would like to thank the referee for very helpful suggestions and correcting a mistake in an earlier version.

\section{References}

[Andersson and Chruściel 1996] L. Andersson and P. T. Chruściel, "Solutions of the constraint equations in general relativity satisfying "hyperboloidal boundary conditions"”, Dissertationes Math. (Rozprawy Mat.) 355 (1996), 100. MR 97e:58217 Zbl 0873.35101

[Chruściel et al. 2005] P. T. Chruściel, E. Delay, J. M. Lee, and D. N. Skinner, "Boundary regularity of conformally compact Einstein metrics”, J. Differential Geom. 69:1 (2005), 111-136. MR 2007g:53042 Zbl 1088.53031

[Eells and Sampson 1964] J. Eells, Jr. and J. H. Sampson, "Harmonic mappings of Riemannian manifolds", Amer. J. Math. 86 (1964), 109-160. MR 29 \#1603 Zbl 0122.40102 
[Graham 1983] C. R. Graham, "The Dirichlet problem for the Bergman Laplacian. I", Comm. Partial Differential Equations 8:5 (1983), 433-476; II, ibid., 563-641. MR 85b:35019 Zbl 0527.35031

[Hartman 1967] P. Hartman, “On homotopic harmonic maps”, Canad. J. Math. 19 (1967), 673-687. MR 35 \#4856 Zbl 0148.42404

[Hörmander 1983] L. Hörmander, The analysis of linear partial differential operators I., vol. 256, Grundlehren der Mathematischen Wissenschaften, Springer, Berlin, 1983. MR 717035

[Kichenassamy 2004] S. Kichenassamy, "On a conjecture of Fefferman and Graham", Adv. Math. 184:2 (2004), 268-288. MR 2005e:53051 Zbl 1066.53083

[Lee 2006] J. M. Lee, "Fredholm operators and Einstein metrics on conformally compact manifolds”, Mem. Amer. Math. Soc. 183:864 (2006), 83. MR 2007m:53047 Zbl 1112.53002

[Li and Tam 1991] P. Li and L.-F. Tam, "The heat equation and harmonic maps of complete manifolds", Invent. Math. 105:1 (1991), 1-46. MR 93e:58039 Zbl 0748.58006

[Li and Tam 1993a] P. Li and L.-F. Tam, "Uniqueness and regularity of proper harmonic maps", Ann. of Math. (2) 137:1 (1993), 167-201. MR 93m:58027 Zbl 0776.58010

[Li and Tam 1993b] P. Li and L.-F. Tam, "Uniqueness and regularity of proper harmonic maps. II", Indiana Univ. Math. J. 42:2 (1993), 591-635. MR 94i:58044 Zbl 0790.58011

[Liao and Tam 1992] G. G. Liao and L.-F. Tam, "On the heat equation for harmonic maps from noncompact manifolds", Pacific J. Math. 153:1 (1992), 129-145. MR 93b:58040 Zbl 0723.58017

[Schoen and Yau 1979] R. Schoen and S. T. Yau, "Compact group actions and the topology of manifolds with nonpositive curvature”, Topology 18:4 (1979), 361-380. MR 81a:53044 Zbl 0424.58012

Received July 31, 2006. Revised February 25, 2007.

HAO YIN

Mathematics Department

EAST CHINA NORMAL UNIVERSITY

NORTH ZHONGSHAN ROAD, 3663

SHANGHAI, 200062

CHINA

haoyin@sjtu.edu.cn 